\title{
Vanishing theorems of the basic harmonic forms on a complete foliated Riemannian manifold
}

\author{
Seoung Dal Jung and Huili Liu
}

\begin{abstract}
It is well-known that on a compact foliated Riemannian manifold $(M, \mathcal{F})$ with some transversal curvature conditions, there are no nontrivial basic harmonic $r(0<r<q=\operatorname{codim} \mathcal{F})$-forms ( M. Min-Oo et al., J. Reine Angew. Math. 415 (1991) 9). In this paper, we extend the above facts to a complete foliated Riemannian manifold.
\end{abstract}

\section{Introduction}

Let $(M, g, \mathcal{F})$ be a foliated Riemannian manifold with a foliation $\mathcal{F}$ and a bundlelike metric $g$ with respect to $\mathcal{F}$. A foliated Riemannian manifold is a Riemannian manifold with a Riemannian foliation, i.e., a foliation on a smooth manifold such that the normal bundle is endowed with a metric whose Lie derivative is zero along leaf directions (see [13). A Riemannian metric on $M$ is bundle-like if the leaves of the foliation $\mathcal{F}$ are locally equidistant, that is, the metric $g$ on $M$ induces a holonomy invariant transverse metric on the normal bundle $Q=T M / T \mathcal{F}$, where $T \mathcal{F}$ is the tangent bundle of $\mathcal{F}$. Every Riemannian foliation admits bundle-like metrics. Many researchers have studided basic forms and the basic Laplacian on foliated Riemannian manifolds. Basic forms are locally forms on the space of leaves; that is, forms $\phi$ satisfying $i(X) \phi=i(X) d \phi=0$ for all $X \in T \mathcal{F}$. Basic forms are preserved by the exterior derivative and are used to define basic de-Rham cohomology groups $H_{B}^{*}(\mathcal{F})$. The basic Laplacian $\Delta_{B}$ for a given bundlelike metric is a version of the Laplace operator that preserves the basic forms. It is well-known 6, 6] that on a closed oriented manifold $M$ with a transversally oriented Riemannian foliation $\mathcal{F}, H_{B}^{r}(\mathcal{F}) \cong \mathcal{H}_{B}^{r}(\mathcal{F})$, where $\mathcal{H}_{B}^{r}(\mathcal{F})=\operatorname{ker} \Delta_{B}$ is finite dimensional. And so $\chi_{B}(\mathcal{F})=\sum_{r=0}^{q}(-1)^{r} \operatorname{dim} \mathcal{H}_{B}^{r}(\mathcal{F})$, where $\chi_{B}(\mathcal{F})$ is the basic Euler characteristic [2. In 1991, M. Min-Oo et al. 9 proved that on a closed foliated Riemannian manifold $M$, if the transversal curvature operator of

2000 Mathematics Subject Classification. 53C12, 58E20

Key words and phrases. Basic harmonic forms, Basic Laplacian 
$\mathcal{F}$ is positive definite, then $H_{B}^{r}(\mathcal{F})=0(0<r<q)$, that is, any basic harmonic $r$-form is trivial.

In this paper, we study the basic $r$-forms on a complete foliated Riemannian manifold.

Main Theorem. Let $(M, g, \mathcal{F})$ be a complete foliated Riemannian manifold and all leaves be compact. Assume that the mean curvature form is bounded and coclosed.

(1) If the transversal Ricci cuvature of $\mathcal{F}$ is positive-definite, then any $L^{2}$-basic harmonic 1-forms $\phi$ with $\phi \in \mathcal{S}_{B}$ are trivial.

(2) If the curvature endomorphism of $\mathcal{F}$ is positive-definite, then any $L^{2}$-basic harmonic $r$-forms $\phi$ with $\phi \in \mathcal{S}_{B}$ are trivial.

Here $\mathcal{S}_{B}$ is the Sobolev space of basic forms whose derivative belong to $L^{2} \Omega_{B}^{*}(\mathcal{F})$.

Note that in 1980, H. Kitahara 8 proved that under the same condition of the transversal Ricci curvature, there are no nontrivial basic $\Delta_{T}$-harmonic 1-forms with finite global norms. Here $\Delta_{T}$ is a different operator to the basic Laplacian $\Delta_{B}$. If $\mathcal{F}$ is minimal, then $\Delta_{T}=\Delta_{B}$.

\section{Preliminaries}

Let $(M, g, \mathcal{F})$ be a $(p+q)$-dimensional complete foliated Riemannian manifold with a foliation $\mathcal{F}$ of codimension $q$ and a bundle-like metric $g$ with respect to $\mathcal{F}$. Let $T M$ be the tangent bundle of $M, T \mathcal{F}$ its integrable subbundle given by $\mathcal{F}$, and $Q=T M / T \mathcal{F}$ the corresponding normal bundle of $\mathcal{F}$. Then we have an exact sequence of vector bundles

$$
0 \longrightarrow T \mathcal{F} \longrightarrow T M \underset{\sigma}{\stackrel{\pi}{\leftrightarrows}} Q \longrightarrow 0,
$$

where $\pi: T M \rightarrow Q$ is a projection and $\sigma: Q \rightarrow T \mathcal{F}^{\perp}$ is a bundle map satisfying $\pi \circ \sigma=i d$. Let $g_{Q}$ be the holonomy invariant metric on $Q$ induced by $g$, i.e., $\theta(X) g_{Q}=0$ for any vector field $X \in T \mathcal{F}$, where $\theta(X)$ is the transverse Lie derivative $\left[\right.$. Let $R^{Q}$ and $\operatorname{Ric}^{Q}$ be the transversal curvature tensor and transversal Ricci operator of $\mathcal{F}$ with respect to the transversal Levi-Civita connection $\nabla^{Q} \equiv \nabla$ in $Q$ [14, respectively. A differential form $\phi \in \Omega^{r}(M)$ is basic if $i(X) \phi=0$ and $i(X) d \phi=0$ for all $X \in T \mathcal{F}$. In a distinguished chart $\left(x_{1}, \cdots, x_{p} ; y_{1}, \cdots, y_{q}\right)$ of 
$\mathcal{F}$, a basic $r$-form $\phi$ is expressed by

$$
\phi=\sum_{a_{1}<\cdots<a_{r}} \phi_{a_{1} \cdots a_{r}} d y_{a_{1}} \wedge \cdots \wedge d y_{a_{r}}
$$

where the functions $\phi_{a_{1} \cdots a_{r}}$ are independent of $x$. Let $\Omega_{B}^{r}(\mathcal{F})$ be the set of all basic $r$-forms on $M$. Then $\Omega^{r}(M)=\Omega_{B}^{r}(\mathcal{F}) \oplus \Omega_{B}^{r}(\mathcal{F})^{\perp}$ [1]. Now, we recall the star operator $\bar{*}: \Omega_{B}^{r}(\mathcal{F}) \rightarrow \Omega_{B}^{q-r}(\mathcal{F})$ given by [1]

$$
\bar{*} \phi=(-1)^{p(q-r)} *\left(\phi \wedge \chi_{\mathcal{F}}\right), \quad \forall \phi \in \Omega_{B}^{r}(\mathcal{F}),
$$

where $\chi_{\mathcal{F}}$ is the characteristic form of $\mathcal{F}$ and $*$ is the Hodge star operator associated to $g$. For any basic forms $\phi, \psi \in \Omega_{B}^{r}(\mathcal{F})$, it is well-known [12 that $\phi \wedge \bar{*} \psi=\psi \wedge \bar{*} \phi$ and $\bar{*}^{2} \phi=(-1)^{r(q-r)} \phi$. The operator $d_{B}$ is the restriction of $d$ to the basic forms, i.e., $d_{B}=\left.d\right|_{\Omega_{B}^{*}(\mathcal{F})}$. Let $d_{T}=d_{B}-\kappa_{B} \wedge$ and $\delta_{T}=(-1)^{q(r+1)+1} \bar{*} d_{B} \bar{*}$, where $\kappa_{B}$ is the basic part of the mean curvature form $\kappa$ of $\mathcal{F}$ [1]. Note that $\kappa_{B}$ is closed, i.e., $d \kappa_{B}=0$ [10]14]. The operator $\delta_{B}: \Omega_{B}^{r}(\mathcal{F}) \rightarrow \Omega_{B}^{r-1}(\mathcal{F})$ is defined by

$$
\delta_{B} \phi=(-1)^{q(r+1)+1} \bar{*} d_{T} \bar{*} \phi=\delta_{T} \phi+i\left(\kappa_{B}^{\sharp}\right) \phi,
$$

where $(\cdot)^{\sharp}$ is the $g_{Q}$-dual vector field of $(\cdot)$. Generally, $\delta_{B}$ is not a restriction of $\delta$ on $\Omega_{B}^{r}(\mathcal{F})$, i.e., $\delta_{B} \neq\left.\delta\right|_{\Omega_{B}^{r}(\mathcal{F})}$, where $\delta$ is the formal adjoint of $d$. But $\delta_{B} \omega=\delta \phi$ for any basic 1-form $\phi$. Let $\Delta_{B}=d_{B} \delta_{B}+\delta_{B} d_{B}$ be a basic Laplacian. Then $\left.\Delta^{M}\right|_{\Omega_{B}^{0}(\mathcal{F})}=\Delta_{B}$ [6], where $\Delta^{M}$ is the Laplacian on $M$. Let $\left\{E_{a}\right\}(a=1, \cdots, q)$ be a local orthonormal basic frame of $Q$ and $\theta^{a}$ a $g_{Q}$-dual 1-form to $E_{a}$. We define $\nabla_{\mathrm{tr}}^{*} \nabla_{\mathrm{tr}}: \Omega_{B}^{r}(\mathcal{F}) \rightarrow \Omega_{B}^{r}(\mathcal{F})$ by

$$
\nabla_{\mathrm{tr}}^{*} \nabla_{\mathrm{tr}}=-\sum_{a} \nabla_{E_{a}, E_{a}}^{2}+\nabla_{\kappa_{B}^{\sharp}}
$$

where $\nabla_{X, Y}^{2}=\nabla_{X} \nabla_{Y}-\nabla_{\nabla_{X}^{M} Y}$ for any $X, Y \in T M$ and $\nabla^{M}$ is the Levi-Civita connection with respect to $g$. Then the generalized Weitzenböck type formula on $\Omega_{B}^{r}(\mathcal{F})$ is given by [4]

$$
\Delta_{B} \phi=\nabla_{\mathrm{tr}}^{*} \nabla_{\mathrm{tr}} \phi+F(\phi)+A_{\kappa_{B}^{\sharp}} \phi
$$

for any $\phi \in \Omega_{B}^{r}(\mathcal{F})$, where $F=\sum_{a, b=1}^{q} \theta^{a} \wedge i\left(E_{b}\right) R^{Q}\left(E_{b}, E_{a}\right)$ and

$$
A_{Y} \phi=\theta(Y) \phi-\nabla_{Y} \phi
$$


In particular, for a 1 -form $\phi, F(\phi)^{\sharp}=\operatorname{Ric}^{Q}\left(\phi^{\sharp}\right)$ and $A_{Y} s=-\nabla_{\sigma(s)} \pi(Y)$. Let $\Omega_{B, o}^{*}(\mathcal{F})$ be the space of basic forms with compact supports.

Let $\nu$ be the transversal volume form, i.e., $* \nu=\chi_{\mathcal{F}}$. The pointwise inner product $\langle\cdot, \cdot\rangle$ on $\Omega_{B}^{r}(\mathcal{F})$ is given by

$$
\langle\phi, \psi\rangle \nu=\phi \wedge \bar{*} \psi
$$

for any basic forms $\phi, \psi \in \Omega_{B}^{r}(\mathcal{F})$. And the global inner product $\ll \cdot, \cdot \gg$ on $\Omega_{B}^{*}(\mathcal{F})$ is defined by

$$
\ll \phi, \psi \gg=\int_{M}\langle\phi, \psi\rangle \mu_{M}
$$

for any $\phi, \psi \in \Omega_{B}^{r}(\mathcal{F})$, one of which has compact support, where $\mu_{M}=\nu \wedge \chi_{\mathcal{F}}$ is the volume form with respect to $g$. It is well-known [4] that $\ll \nabla_{\mathrm{tr}}^{*} \nabla_{\mathrm{tr}} \phi, \psi \gg=\ll$ $\nabla_{\mathrm{tr}} \phi, \nabla_{\mathrm{tr}} \psi \gg$ for any $\phi, \psi \in \Omega_{B, 0}^{r}(\mathcal{F})$ and

$$
\ll d_{B} \phi, \psi \gg=\ll \phi, \delta_{B} \psi \gg
$$

for any $\phi \in \Omega_{B, o}^{r}(\mathcal{F}), \psi \in \Omega_{B, o}^{r+1}(\mathcal{F})$. The basic form $\phi$ is said to be $L^{2}$-basic form if $\phi$ has finite global norm, i.e., $\|\phi\|^{2}<\infty$. Let $\mathcal{H}_{B, 2}^{r}(\mathcal{F})$ be the space of $L^{2}$-basic harmonic forms, i.e.,

$$
\mathcal{H}_{B, 2}^{r}(\mathcal{F})=\left\{\phi \in L^{2} \Omega_{B}^{r}(\mathcal{F}) \mid d_{B} \phi=\delta_{B} \phi=0\right\} .
$$

Generally, the space $\mathcal{H}_{B, 2}^{r}(\mathcal{F})$ can have infinite dimension. And if the dimension of $\mathcal{H}_{B, 2}^{r}(\mathcal{F})$ is finite, then it depends on the bundle-like metric. Trivially, if $M$ is compact, then $\mathcal{H}_{B, 2}^{r}(\mathcal{F}) \cong H_{B}^{r}(\mathcal{F})$. So we study the vanishing properties of the $L^{2}$-basic harmonic spaces on a complete foliated Riemannian manifold.

Remark 2.1 (1) The operator $\delta_{T}$ is the formal adjoint of $d_{B}$ with respect to the global norm $(\cdot, \cdot)$, which is given by

$$
(\phi, \psi)=\int_{M}\langle\phi, \psi\rangle \nu \wedge d x_{1} \wedge \cdots \wedge d x_{p}
$$

Let $\Delta_{T}=d_{B} \delta_{T}+\delta_{T} d_{B}$ be a Laplacian. If the foliation is minimal, then $\delta_{T}=\delta_{B}$. So $\Delta_{B}=\Delta_{T}$.

(2) In 1980, H. Kitahara 8 proved that if the transversal Ricci curvature is nonnegative and positive at some point, then there are no nontrivial $L^{2}$-basic $\Delta_{T}$-harmonic 1-forms. 


\section{Vanishing theorem}

Let $(M, g, \mathcal{F})$ be a complete foliated Riemannian manifold with a foliation $\mathcal{F}$ of codimension $q$ and a bundle-like metric $g$ with respect to $\mathcal{F}$. Assume that all leaves of $\mathcal{F}$ are compact. Now, we consider a smooth function $\mu$ on $\mathbb{R}$ satisfying

(i) $0 \leq \mu(t) \leq 1$ on $\mathbb{R}, \quad($ ii $) \mu(t)=1$ for $t \leq 1, \quad($ iii $) \mu(t)=0$ for $t \geq 2$.

Let $x_{0}$ be a point in $M$. For each point $y \in M$, we denote by $\rho(y)$ the distance between leaves through $x_{0}$ and $y$. For any real number $l>0$, we define a Lipschitz continuous function $\omega_{l}$ on $M$ by

$$
\omega_{l}(y)=\mu(\rho(y) / l)
$$

Trivially, $\omega_{l}$ is a basic function. Let $B(l)=\{y \in M \mid \rho(y) \leq l\}$ for $l>0$. Then $\omega_{l}$ satisfies the following properties:

$$
\begin{array}{ll}
0 \leq \omega_{l}(y) \leq 1 & \text { for any } y \in M \\
\operatorname{supp} \omega_{l} \subset B(2 l) & \\
\omega_{l}(y)=1 & \text { for any } y \in B(l) \\
\lim _{l \rightarrow \infty} \omega_{l}=1 & \\
\left|d_{B} \omega_{l}\right| \leq \frac{C}{l} & \text { almost everywhere on } M
\end{array}
$$

where $C$ is a positive constant independent of $l$ [15. Hence $\omega_{l} \psi$ has compact support for any basic form $\psi \in \Omega_{B}^{*}(\mathcal{F})$ and $\omega_{l} \psi \rightarrow \psi$ (strongly) when $l \rightarrow \infty$.

Lemma 3.1 [7] For any $\phi \in \Omega_{B}^{r}(\mathcal{F})$, there exists a number $A$ depending only on $\mu$, such that

$$
\begin{aligned}
& \left\|d_{B} \omega_{l} \wedge \phi\right\|_{B(2 l)}^{2} \leq \frac{q A^{2}}{l^{2}}\|\phi\|_{B(2 l)}^{2}, \\
& \left\|d_{B} \omega_{l} \wedge \bar{*} \phi\right\|_{B(2 l)}^{2} \leq \frac{q A^{2}}{l^{2}}\|\phi\|_{B(2 l)}^{2}, \\
& \left\|d_{B} \omega_{l} \otimes \phi\right\|_{B(2 l)}^{2} \leq \frac{q A^{2}}{l^{2}}\|\phi\|_{B(2 l)}^{2},
\end{aligned}
$$

where $\|\phi\|_{B(2 l)}^{2}=\ll \phi, \phi \gg_{B(2 l)}=\int_{B(2 l)}\langle\phi, \phi\rangle \mu_{M}$.

Proposition 3.2 For any $L^{2}$-basic form $\psi$, if $\Delta_{B} \psi=0$, then $d_{B} \psi=0$ and $\delta_{B} \psi=0$. 
Proof. Let $\psi$ be a $L^{2}$-basic form. Then we have

$$
\ll \Delta_{B} \psi, \omega_{l}^{2} \psi \gg_{B(2 l)}=\ll d_{B} \psi, d_{B}\left(\omega_{l}^{2} \psi\right) \gg_{B(2 l)}+\ll \delta_{B} \psi, \delta_{B}\left(\omega_{l}^{2} \psi\right) \gg_{B(2 l)} .
$$

By a direct calculation, we have

$$
\begin{aligned}
d_{B}\left(\omega_{l}^{2} \psi\right) & =\omega_{l}^{2} d_{B} \psi+2 \omega_{l} d_{B} \omega_{l} \wedge \psi, \\
\delta_{B}\left(\omega_{l}^{2} \psi\right) & =\omega_{l}^{2} \delta_{B} \psi+(-1)^{q(r+1)+1} \bar{*}\left(2 \omega_{l} d_{B} \omega_{l} \wedge \bar{*} \psi\right) .
\end{aligned}
$$

From (3.1), (3.2) and (3.3), if $\Delta_{B} \psi=0$, then

$$
\begin{aligned}
& \left\|\omega_{l} d_{B} \psi\right\|_{B(2 l)}^{2}+\left\|\omega_{l} \delta_{B} \psi\right\|_{B(2 l)}^{2} \\
& =-2 \ll \omega_{l} d_{B} \psi, d_{B} \omega_{l} \wedge \psi \gg_{B(2 l)}+2(-1)^{q(r+1)} \ll \omega_{l} \delta_{B} \psi, \bar{*}\left(d_{B} \omega_{l} \wedge \bar{*} \psi\right) \gg_{B(2 l)} .
\end{aligned}
$$

Hence by the the Schwartz's inequality and Lemma 3.1, we have

$$
\begin{aligned}
& \left\|\omega_{l} d_{B} \psi\right\|_{B(2 l)}^{2}+\left\|\omega_{l} \delta_{B} \psi\right\|_{B(2 l)}^{2} \\
& \leq \epsilon_{1}\left\|\omega_{l} d_{B} \psi\right\|_{B(2 l)}^{2}+\epsilon_{2}\left\|\omega_{l} \delta_{B} \psi\right\|_{B(2 l)}^{2}+\frac{B_{1}}{l}\|\psi\|_{B(2 l)}^{2}
\end{aligned}
$$

for some positive real numbers $\epsilon_{1}, \epsilon_{2}$ and $B_{1}$. Therefore, we have

$$
\left\|\omega_{l} d_{B} \psi\right\|_{B(2 l)}^{2}+\left\|\omega_{l} \delta_{B} \psi\right\|_{B(2 l)}^{2} \leq \frac{B_{2}}{l}\|\psi\|_{B(2 l)}^{2}
$$

for some positive real number $B_{2}$. Since $\psi$ is the $L^{2}$-basic form, letting $l \rightarrow \infty$, $d_{B} \psi=\delta_{B} \psi=0$.

Remark 3.3 In 1979, H. Kitahara 7 proved the corresponding result with the Laplacian $\Delta_{T}$. Namely, on a complete foliated manifold, if $\Delta_{T} \phi=0$, then $d_{B} \phi=$ $\delta_{T} \phi=0$.

Now we prove the vanishing theorem of the $L^{2}$-basic harmonic form on a complete foliated Riemannian manifold. First of all, we prepare some lemmas.

Lemma 3.4 Let $(M, g, \mathcal{F})$ be a complete foliated Riemannian manifold whose leaves are compact. Suppose that $\kappa_{B}$ is bounded and coclosed. Then for any $L^{2}$-basic harmonic form $\phi$,

$$
\limsup _{l \rightarrow \infty} \ll A_{\kappa_{B}^{\sharp}} \phi, \omega_{l}^{2} \phi \gg_{B(2 l)}=0 .
$$


Proof. Let $\phi$ be a $L^{2}$-basic harmonic form. Since $\theta(X) \phi=d_{B} i(X) \phi$, from (2.6) we have

$$
\ll A_{\kappa_{B}^{\sharp}} \phi, \omega_{l}^{2} \phi \gg_{B(2 l)}=\ll d_{B} i\left(\kappa_{B}^{\sharp}\right) \phi, \omega_{l}^{2} \phi \gg_{B(2 l)}-\ll \nabla_{\kappa_{B}^{\sharp}} \phi, \omega_{l}^{2} \phi \gg_{B(2 l)}
$$

Since $\delta_{B} \phi=0$, from (3.3) and Lemma 3.1, we have

$$
\begin{aligned}
\left|\ll d_{B} i\left(\kappa_{B}^{\sharp}\right) \phi, \omega_{l}^{2} \phi \gg_{B(2 l)}\right| & =2\left|\ll \omega_{l} i\left(\kappa_{B}^{\sharp}\right) \phi, \bar{*}\left(d_{B} \omega_{l} \wedge \bar{*} \phi\right) \gg_{B(2 l)}\right| \\
& \leq \epsilon_{3}\left\|\omega_{l} i\left(\kappa_{B}^{\sharp}\right) \phi\right\|_{B(2 l)}^{2}+\frac{1}{\epsilon_{3}}\left\|d_{B} \omega_{l} \wedge \bar{*} \phi\right\|_{B(2 l)}^{2} \\
& \leq \epsilon_{3}\left\|\omega_{l} i\left(\kappa_{B}^{\sharp}\right) \phi\right\|_{B(2 l)}^{2}+\frac{B_{3}}{l^{2}}\|\phi\|_{B(2 l)}^{2}
\end{aligned}
$$

for some positive real numbers $\epsilon_{3}$ and $B_{3}$. By using $\left|i\left(\kappa_{B}^{\sharp}\right) \phi\right|^{2}+\left|\kappa_{B} \wedge \phi\right|^{2}=$ $\left|\kappa_{B}\right|^{2}|\phi|^{2}$, we have

$$
\left|\ll d_{B} i\left(\kappa_{B}^{\sharp}\right) \phi, \omega_{l}^{2} \phi \gg_{B(2 l)}\right| \leq \epsilon_{3} \max \left(\left|\kappa_{B}\right|^{2}\right)\left\|\omega_{l} \phi\right\|_{B(2 l)}^{2}+\frac{B_{3}}{l^{2}}\|\phi\|_{B(2 l)}^{2}
$$

On the other hand, since $\delta_{B} \kappa_{B}=0$, by a direct calculation, we have

$$
\begin{aligned}
\ll \nabla_{\kappa_{B}^{\sharp}} \phi, \omega_{l}^{2} \phi \gg_{B(2 l)} & =\frac{1}{2} \ll d_{B}\left(\left|\omega_{l} \phi\right|^{2}\right), \kappa_{B} \gg_{B(2 l)}-\ll \omega_{l} \phi, \kappa_{B}^{\sharp}\left(\omega_{l}\right) \phi \gg_{B(2 l)} \\
& =-\ll \omega_{l} \phi, \kappa_{B}^{\sharp}\left(\omega_{l}\right) \phi \gg_{B(2 l)} .
\end{aligned}
$$

Hence by the Schwartz inequality, we have

$$
\begin{aligned}
\left|\ll \nabla_{\kappa_{B}^{\sharp}} \phi, \omega_{l}^{2} \phi \gg_{B(2 l)}\right| & =\left|\ll \omega_{l} \phi, \kappa_{B}^{\sharp}\left(\omega_{l}\right) \phi \gg_{B(2 l)}\right| \\
& \leq \epsilon_{4}\left\|\omega_{l} \phi\right\|_{B(2 l)}^{2}+\frac{B_{4}}{l^{2}} \max \left(\left|\kappa_{B}\right|^{2}\right)\|\phi\|_{B(2 l)}^{2}
\end{aligned}
$$

for a positive real numbers $\epsilon_{4}$ and $B_{4}$. From (3.6) and (3.7), by letting $l \rightarrow \infty$, we have

$$
\begin{aligned}
& \limsup _{l \rightarrow \infty}\left|\ll d_{B} i\left(\kappa_{B}^{\sharp}\right) \phi, \omega_{l}^{2} \phi \gg_{B(2 l)}\right| \leq \epsilon_{3} \max \left(\left|\kappa_{B}\right|^{2}\right)\|\phi\|^{2}, \\
& \underset{l \rightarrow \infty}{\limsup }\left|\ll \nabla_{\kappa_{B}^{\sharp}} \phi, \omega_{l}^{2} \phi \gg_{B(2 l)}\right| \leq \epsilon_{4}\|\phi\|^{2} .
\end{aligned}
$$

Since $\epsilon_{3}$ and $\epsilon_{4}$ are arbitrary positive numbers, we have

$$
\begin{aligned}
& \limsup _{l \rightarrow \infty}\left|\ll d_{B} i\left(\kappa_{B}^{\sharp}\right) \phi, \omega_{l}^{2} \phi \gg_{B(2 l)}\right|=0, \\
& \limsup _{l \rightarrow \infty}\left|\ll \nabla_{\kappa_{B}^{\sharp}} \phi, \omega_{l}^{2} \phi \gg_{B(2 l)}\right|=0 .
\end{aligned}
$$

Hence from (3.5), (3.8) and (3.9), the proof is completed. 
Theorem 3.5 Let $(M, g, \mathcal{F})$ be as in Lemma 3.4. Suppose that $\kappa_{B}$ is bounded and coclosed. If the curvature endomorphism $F$ of $\mathcal{F}$ is positive-definite, then any $L^{2}$ - basic harmonic $r$-forms $\phi$ with $\phi \in \mathcal{S}_{B}$ are trivial, i.e., $\mathcal{H}_{B, 2}^{r}(\mathcal{F})=\{0\}$.

Proof. Let $\phi$ be a $L^{2}$-basic harmonic $r$-form. From (2.5) and Proposition 3.2, we have

$$
\left\langle\nabla_{\mathrm{tr}}^{*} \nabla_{\mathrm{tr}} \phi, \omega_{l}^{2} \phi\right\rangle+\left\langle F(\phi), \omega_{l}^{2} \phi\right\rangle+\left\langle A_{\kappa_{B}^{\sharp}} \phi, \omega_{l}^{2} \phi\right\rangle=0 .
$$

On the other hand, a direct calculation gives

$$
\ll \nabla_{\text {tr }}^{*} \nabla_{\text {tr }} \phi, \omega_{l}^{2} \phi \gg_{B(2 l)}=\ll \nabla_{\text {tr }} \phi, 2 \omega_{l} d_{B} \omega_{l} \otimes \phi \gg_{B(2 l)}+\left\|\omega_{l} \nabla_{\text {tr }} \phi\right\|_{B(2 l)}^{2} .
$$

From Lemma 3.1, we have

$$
\left|\ll \nabla_{\mathrm{tr}} \phi, 2 \omega_{l} d_{B} \omega_{l} \otimes \phi \gg_{B(2 l)}\right| \leq \epsilon_{5}\left\|\omega_{l} \nabla_{\mathrm{tr}} \phi\right\|_{B(2 l)}^{2}+\frac{B_{5}}{l^{2}}\|\phi\|_{B(2 l)}^{2}
$$

for some positive constants $\epsilon_{5}$ and $B_{5}$. Hence by letting $l \rightarrow \infty$, we have

$$
\limsup _{l \rightarrow \infty} \ll \nabla_{\operatorname{tr}} \phi, 2 \omega_{l} d_{B} \omega_{l} \otimes \phi \gg_{B(2 l)} \leq \epsilon_{5}\left\|\nabla_{\operatorname{tr}} \phi\right\|^{2} .
$$

Since $\epsilon_{5}$ is arbitrary and $\phi \in \mathcal{S}_{B}$ ( i.e., $\left\|\nabla_{\operatorname{tr}} \phi\right\|^{2}<\infty$ ), we have

$$
\limsup _{l \rightarrow \infty} \ll \nabla_{\operatorname{tr}} \phi, 2 \omega_{l} d_{B} \omega_{l} \otimes \phi \gg_{B(2 l)}=0 .
$$

Hence from (3.11), (3.12) and Lemma 3.4, we have

$$
\left\|\nabla_{\mathrm{tr}} \phi\right\|^{2}+\limsup _{l \rightarrow \infty} \ll F(\phi), \omega_{l}^{2} \phi \gg_{B(2 l)}=0,
$$

which complete the proof.

Since $F\left(\phi^{\sharp}\right)=\operatorname{Ric}^{Q}\left(\phi^{\sharp}\right)$ for any basic 1-form $\phi$, we have the following corollary.

Corollary 3.6 Let $(M, g, \mathcal{F})$ be as in Lemma 3.4. Suppose that $\kappa_{B}$ is bounded and coclosed. If the transversal Ricci curvature $\mathrm{Ric}^{Q}$ is positive-definite, then any $L^{2}$-basic harmonic 1-forms $\phi$ with $\phi \in \mathcal{S}_{B}$ are trivial, $\mathcal{H}_{B, 2}^{1}(\mathcal{F})=\{0\}$.

Acknowledgements. The first author was supported by the National Research Foundation of Korea(NRF) grant funded by the Korea government (MSIP) (NRF2015R1A2A2A01003491) and the second author was supported by NSFC (No. 11371080). 


\section{References}

[1] J. A. Alvarez López, The basic component of the mean curvature of Riemannian foliations, Ann. Global Anal. Geom. 10 (1992), 179-194.

[2] V. Blefi, E. Park and K. Richardson, A Hopf index theorem for foliations, Diff. Geom. Appl. 18 (2003), 319-341.

[3] J. J. Hebda, Curvature and focal points in Riemannian foliations, Indiana Univ. Math. J. 35 (1986), 321-331.

[4] S. D. Jung, The first eigenvalue of the transversal Dirac operator, J. Geom. Phys. 39 (2001), 253-264.

[5] F. W. Kamber and Ph. Tondeur, Infinitesimal automorphisms and second variation of the energy for harmonic foliations, Tôhoku Math. J. 34 (1982), 525-538.

[6] F. W. Kamber and Ph. Tondeur, De Rham-Hodge theory for Riemannian foliations, Math. Ann. 277 (1987), 415-431.

[7] H. Kitahara, Remarks on square-integrable basic cohomology spaces on a foliated Riemannian manifold, Kodai Math. J. 2 (1979), 187-193.

[8] H. Kitahara, Nonexistence of nontrivial $\square$ "-harmonic 1-forms on a complete foliated Riemannian manifold, Trans. Amer. Math. Soc. 262 (1980), 429-435.

[9] M. Min-Oo, E. Ruh and Ph. Tondeur, Vanishing theorems for the basic cohomology of Riemannian foliations, J. Reine Angew. Math. 415 (1991), 167-174.

[10] J. S. Pak and S. D. Jung, A transversal Dirac operator and some vainshing theorems on a complete foliated Riemannian manifold, Math. J. Toyama Univ. 16 (1993), 97-108.

[11] J. S. Pak and S. D. Jung, Some vanishing theorems on complete Kähler foliations, Acta Math. Hungar. 77 (1997), 15-28.

[12] E. Park and K. Richardson, The basic Laplacian of a Riemannian foliation, Amer. J. Math. 118 (1996), 1249-1275.

[13] B. Reinhart, Differential Geometry of Foliations, Springer-Verlag, 1983. 
[14] Ph. Tondeur, Geometry of foliations, Basel: Birkhäuser Verlag, 1997.

[15] S. Yorozu, Notes on suare-integrable cohomology spaces on certain foliated manifolds, Trans. Amer. Math. Soc. 255 (1979), 329-341.

Department of Mathematics and Research Institute for Basic Sciences, Jeju National University, Jeju 690-756, Korea

E-mail address : sdjung@jejunu.ac.kr

Department of Mathematics, Northeastern University, Shenyang 110004, P. R. China

E-mail address : liuhl@mail.neu.edu.cn 